$$
\begin{gathered}
\text { 수업평가에 대한 교수와 학생 인식 비교 } \\
- \text { 일개 의과대학 사례 - } \\
\text { 아주대학교 의과대학 의학교육실 } \\
\text { 채 수 진 · 임 기 영 }
\end{gathered}
$$

$=$ Abstract $=$

\title{
A Comparison of Student and Faculty Perspectives on Course Evaluation in a Medical School
}

\author{
Su-Jin Chae, EdD, Ki-Young Lim, MD, $\mathrm{PhD}$. \\ Office of Medical Education, Ajou University School of Medicine, Suwon, Korea
}

\begin{abstract}
Purpose: The purpose of this study was to analyze the differences in perception between the students and faculty on course evaluation by the students and to pursue the improving of the course evaluation system.

Methods: This study targeted 61 faculty and 88 students of the School of Medicine at A University. A questionnaire survey was administered.

Results: Both the students and faculty members agreed with the need of a course evaluation system, but there were differences in perception between the two groups in terms of efficiency and factors affecting evaluation results.

Conclusion: The department in charge of course evaluations of the school of medicine should review the differences in perception between students and faculty and use the reviewed content to improve the present course evaluation system.
\end{abstract}

Key Words: Course evaluation, Faculty evaluation

\section{서 론}

2000년부터 도입된 의과대학 인정평가와 같은 현 실적인 문제와 맞물려 2007년 현재 의학전문대학원 을 포함한 국내 41 개 의과대학 모두가 수업평가를 실시하고 있다. 의과대학에서 '학생에 의한 수업평
가제도'는 90년대 초반에 수업의 질을 향상시킬 것 을 목적으로 시작하여 최근에는 일부의 대학에서 교수들의 성과급이나 승진 및 재임용의 평가 지표 로까지 활용되고 있다 (Chae \& Lim, 2007).

국내의 한 조사에 의하면 (Hong, 2006) 수업평가 는 거의 모든 학교에서 실시되고 있는데 반해서 학

교신저자: 채수진, 아주대학교 의과대학 의학교육실, 경기도 수원시 영통구 원천동 산5번지

Tel: 031)219-4465, Fax: 031)219-5179, E-mail: edujin@ajou.ac.kr 
수업평가에 대한 교수와 학생 인식 비교 - 일개 의과대학 사례 -

Table I. The Opinions of Student and Faculty Regarding the Necessity and Effectiveness of Course Evaluation $\mathrm{N}(\%)$

\begin{tabular}{ccccccc}
\hline \hline & Group & Strongly agree & Agree & Disagree & Strongly disagree & I don't know \\
\hline \multirow{2}{*}{$1^{*}$} & Student & $30(34.1)$ & $44(50)$ & $6(6.8)$ & $1(1.1)$ & $7(8.0)$ \\
& Faculty & $12(19.7)$ & $28(45.9)$ & $1(1.6)$ & 0 & $20(32.8)$ \\
\multirow{2}{*}{$2^{\dagger}$} & Student & $4(4.5)$ & $26(29.5)$ & $39(44.3)$ & $16(18.2)$ & $3(3.4)$ \\
& Faculty & $6(9.8)$ & $33(54.1)$ & $18(29.5)$ & $2(3.3)$ & $2(3.3)$ \\
\multirow{2}{*}{$3^{\ddagger}$} & Student & $6(6.8)$ & $52(59.1)$ & $18(20.5)$ & $2(2.3)$ & $8(9.1)$ \\
& Faculty & $5(8.2)$ & $31(50.8)$ & $21(34.4)$ & $2(3.3)$ & $2(3.3)$ \\
\hline
\end{tabular}

* Do you think course evaluations are needed?, ${ }^{\dagger}$ Have course evaluation results affected teaching method of individual tutor?, ${ }^{\ddagger}$ Have course evaluation results improved the overall course quality?

생과 교수는 여전히 그 실효성이나 타당성, 신뢰성 등에 대해서 확신을 하지 못하는 것으로 나타났다. $34 \%$ 의 학생이 '질문은 읽지 않은 채 버튼만 대충 누른다'고 응답하여 불성실함을 나타냈고, 응답자의 $69 \%$ 가 ‘수업평가 후 학생들의 평가내용이 학교 또 는 교수에게 반영되지 않는 것 같다’라고 수업평가 에 대한 불신을 드러냈다고 한다.

본 연구는 수업평가제도의 유용성에 대한 물음에 서 출발한다. 수업평가제도에 대한 역사가 길지 않 은 의과대학의 경우 교수와 학생들은 수업평가제도 를 찬성하는가? 수업평가를 반대한다면 그 이유가 무엇인가? 수업평가 결과에 미치는 요인이 무엇이 며, 수업평가 내용으로 중요하게 생각하는 항목은 무엇인가?

본 연구는 의과대학에서 시행하고 있는 수업평가 에 대해서 학생과 교수의 인식의 차이점을 찾아내 고 이를 바탕으로 의과대학 수업평가의 개선 방안 을 모색하는 데에 목적이 있다.

\section{대상 및 방법}

$\mathrm{A}$ 의과대학 220 명의 전체 교수 중 2007년 9월 교 수회의에 참석한 113 명 교수와 의학과 2학년 (52명) 과 3학년 (49명) 학생들에게 설문조사를 실시하였 다. 113 명의 교수 중 설문에 응답한 교수는 61 명으 로 54\%의 회수율을 보였으며, 2학년은 83\% (43명),
3학년은 96\% (49명)의 회수율을 나타냈다.

$\mathrm{A}$ 의과대학은 2006학년부터 의과대학 공통설문지 를 이용하여 의예과와 의학과를 포함한 전체 학년 을 대상으로 수업평가 및 교수개인별 평가를 실시 하고 있다. $\mathrm{A}$ 의과대학은 의과대학만의 독특한 교육 과정의 운영 및 학사 체계로 인해 대학본부에서 학 기말에 실시하는 온라인 수업평가제도를 따르지 않 고 의과대학에서 별도의 설문지를 개발하여 실시하 고 있다. 수업평가 결과는 학생들의 성적이 모두 입 력된 뒤 각 교실의 주임교수에게 전달되며, 강의를 담당했던 교수 (또는 강사) 개인은 각 교실의 주임교 수를 통해서만 수업평가 결과를 통보받을 수 있다.

설문은 수업평가의 필요성, 효과성, 내용의 중요 성, 수업평가 결과에 미치는 요인 등을 내용으로 하 였으며, 결과는 SPSS통계 프로그램을 사용하여 빈 도분석을 실시하였다.

\section{결 과}

\section{가. 수업평가제도의 필요성과 효과성에 대한 인식}

수업평가제도의 필요성에 대해 학생은 $74 \%$, 교수 는 $65 \%$ 가 찬성한다고 대답함으로써 교수와 학생 모 두 수업평가제도에 대해서 긍정적인 생각을 가지고 있는 것으로 나타났다 (Table I). 수업평가제를 반대 한다면 그 이유가 무엇인지를 물은 결과, 학생들은 수업평가의 결과가 실제로 교수에게 전달되는지 모 
Table II. The Opinions of Student and Faculty on Contents and Factors Affecting Course Evaluation Results

\begin{tabular}{|c|c|c|c|c|}
\hline & Faculty & $\mathrm{n}$ & Student & $\mathrm{n}$ \\
\hline \multirow{4}{*}{$1^{*}$} & Class focused on fun or amusement & 17 & Punctuality & 30 \\
\hline & Degree of difficulty in tests/exams & 15 & Course progress (teaching method) mode & 19 \\
\hline & Lecturing time & 9 & Degree of course difficulty & 19 \\
\hline & No. of participating faculty members & 6 & Degree of difficulty in tests/exams & 5 \\
\hline \multirow{5}{*}{$2^{\dagger}$} & Student's own class attitude evaluation & 26 & $\begin{array}{l}\text { Teaching method and conveying ability } \\
\text { (eliciting student participation, voice and choice } \\
\text { of words) }\end{array}$ & 29 \\
\hline & Lecture level and degree of difficulty & 13 & $\begin{array}{l}\text { Adequacy of teaching tool } \\
\text { (provision of course materials and teaching aids) }\end{array}$ & 16 \\
\hline & Faculty's attitude (enthusiasm, faithfulness) & 8 & Lecture level and degree of difficulty & 16 \\
\hline & Teaching method and conveying ability & 5 & Duplicity degree of learning content & 8 \\
\hline & Test/exam evaluation & 2 & Student's own class attitude evaluation & 1 \\
\hline
\end{tabular}

* Factors affecting course evaluation results, ${ }^{\dagger}$ Contents of course evaluation.

르기 때문에, 평가를 실시해도 수업개선이 이루어지 지 않아서, 교수의 눈치를 보게 되어서 등으로 대답 하였다. 교수가 반대하는 이유로는 인기를 위해서 흥미 위주의 수업을 하게 되고, 학생들이 교과내용 도 모르면서 근거 없는 비방을 한다는 것이 대부분 이었다. 수업태도가 좋지 않은 학생들에게 평가를 받는 것이 불쾌하다고 대답한 교수도 있었다.

수업평가의 효과를 알아보기 위해 강의법 개선 과 수업의 질 개선에 대한 질문을 하였다. 강의법 개선에 대해, 학생은 응답자의 $63 \%$ 가 수업평가 이 후 교수의 강의법 개선이 거의 없다고 대답한 반면, 교수는 $64 \%$ 가 교수 개인의 강의법 개선에 영향을 주었다고 대답함으로써 강의법 개선에 대한 학생과 교수의 인식의 차이가 있었으며, 이는 통계적으로 유의미하게 나타났다 $(\mathrm{p}<.05)$. 수업평가가 수업 전 반의 질을 개선하는 영향을 주었는가에 대해서는 학생과 교수가 각각 $66 \%, 60 \%$ 가 '그렇다'고 대답 하였다.

\section{나. 수업평가 내용과 결과에 미치는 요인에 대한 인식}

Table II는 수업펑가의 결과에 미치는 요인과 수 업평가 항목으로 중요한 내용이 무엇인지를 물은
결과이다. 수업평가 결과에 영향을 미치는 가장 중 요한 요인으로, 학생들은 교수가 수업시간을 엄수 하지 않을 때 아무리 강의를 잘했다고 하더라도 실 제로 낮은 평가를 한다고 대답한 (30명) 반면, 교수 들은 평가가 내용의 중요도와 상관없이 흥미나 재 미에 좌우되는 경향이 있다고 대답하였다 (17명). 학생들은 수업을 제시간에 마치는 교수, 그리고 알 아듣기 쉬운 용어로, 큰 목소리로, 학생의 참여를 유도하는 교수에게 높은 점수를 부여하며 (19명), 수업내용의 난이도 (19명)이나 시험문제의 난이도 $(5$ 명)에 따라 교수를 평가한다고 대답하였다. 교수의 경우도 시험 문제의 난이도 (15명)에 따라 부여받은 평가 점수가 달랐다고 대답하였다. 그러나 교수는 학생들과 달리, 강의시간이 많고 참여교수가 많은 수업일수록 평가점수가 낮은 경향이 있었다고 대답 하였다.

수업평가 항목으로 중요하다고 생각하는 내용을 물은 질문에 대해서, 교수는 학생 자신들의 수업태 도를 스스로 평가할 수 있는 항목 (26명), 강의의 난 이도 또는 이해도 (13명), 교수의 성실도 (8명), 수업 전달의 효율성 (5명), 그리고 학생들이 시험문항을 평가할 수 있는 항목 (2명) 등을 지적하였다. 학생들 
은 학생 참여유도, 목소리의 크기, 학생을 존중하는 말투 등 교수의 수업전달 능력을 수업평가 항목 (29 명)으로 가장 중요하다고 지적하였다 이밖에도 수 업자료를 수업 전에 배포하거나 교재 사용에 대한 내용 (16명), 강의내용의 수준 (16명), 학습내용의 중 복정도 (8명) 등을 중요한 평가 내용으로 꼽았다.

\section{고 찰}

수업평가의 목적은 크게 교수들 스스로가 수업을 더 잘 할 수 있도록 학생들로부터 피드백을 얻기 위한 목적과 대학행정 측면에서 교수의 능력과 기 여도를 점수로 환산하여 승진이나 보수 책정과 같 은 인사 결정에 반영하기 위한 목적으로 구분되며 수업평가는 이 두 가지 목적 중 어느 한쪽으로 치우 쳐 실시되어서는 안 된다는 것에는 이견이 없다 (Chae, 2007). 그럼에도 불구하고 수업평가의 목적 에 맞게 ‘무엇을', '어떻게' 평가해야 하는가라는 물 음에는 끊임없이 문제가 제기되고 있다. 본 연구는 수업평가제도에 대한 학생과 교수의 인식의 차이를 알아보고 개선 방안을 논의하는 데에 목적이 있었 다. 본 연구를 통해 밝혀진 내용과 고찰 내용은 아 래와 같다.

첫째, 교수와 학생 모두 수업평가제도에 대해서는 찬성을 하였지만, 수업평가 점수에 대한 객관성에는 여전히 의문을 가지고 있는 것으로 나타났다. 학생 들은 교수들이 학생 개개인의 수업평가 점수를 알 게 되었을 때의 자신에게 돌아오는 불이익에 대해 서 두려워하였고 심지어 1학년, 2학년 때 실시하였 던 수업평가의 결과가 3학년, 4학년 때 임상실습 성 적에 영향을 미칠 것을 걱정하는 학생도 있었다. 이 것은 수업평가의 객관성에 영향을 줄 수 있는 상당 히 중요한 문제라고 할 수 있다. 이러한 문제를 해 결하기 위해서 수업평가 결과를 교수와 학생 등 의 과대학 이해 관계자 모두에게 알릴 필요가 있으며, 계속적으로 수업의 개선사항과 수업의 질을 체계적 으로 모니터할 필요가 있다.

둘째, 강의법 개선에 관해서 학생과 교수의 의견 차이가 있었지만 수업 전반에 대해서는 두 집단 모
두 개선되었다는 대답을 하였다. 이러한 결과는 학 생들이 '수업의 질 향상'과 '강의법 개선'을 두개의 다른 양상으로 간주하는 데에서 비롯된다. 학생들은 학습내용의 수준이나 양, 학습목표 및 교과내용, 학 습내용의 조직 등의 내용 영역을 수업의 질 향상으 로, 교수-학습 방법, 교수태도 등 방법 영역을 강의 법 개선으로 간주하고 있는 것이다. 이는 학생들이 수업내용의 수준이나 중복 정도보다도 교수의 전달 능력을 수업평가의 중요한 요인으로 대답한 결과와 도 일치한다. 교수와 학생 모두에게 수업평가에 대 한 만족을 주기 위한 방안으로 내용의 영역과 방법 의 영역이 골고루 포함된 수업평가 설문지를 개발 할 필요가 있다.

셋째, 수업평가에 미치는 요인들에 대해서 학생들 은 수업시간 엄수, 수업진행 방식, 수업내용의 난이 도 등의 요인이, 교수들은 흥미 위주의 수업, 시험문 제의 난이도, 강의 시간, 참여 교원의 수 등의 요인 이 수업평가 결과에 영향을 미칠 수 있다고 지적하 였다. 기존 연구 (Kim et al., 2001; Kim, 2005)와 다 른 점은 수업평가에 미치는 요인으로 학생수가 아 닌, 참여 교원수가 지적된 것이다. 이는 팀티칭으로 운영되는 의과대학의 특수성 때문으로 볼 수 있는 데, 수업평가의 결과를 교수업적평가로 활용하는 대 학에서는 이점을 충분히 고려하여 반영해야 할 것 이다.

\section{참 고 문 헌}

Chae, S.J.(2007). A study on course evaluation contents through a written comments analysis of the course evaluation in medical school. The Journal of Yeolin Education, 5(1), 237-255.

Chae, S.J., \& Lim, K.Y.(2007). An analysis of course evaluation programs at Korean medical schools. Korean J Med Educ, 19(2), 133-142.

Hong, K.S.(2006). An analysis of student' response to course evaluation. The Journal of Educational Information and Medi, 12(2), 97-127.

Kim, M.H.(2005). Validity and reliability of lecture 
evaluation. Asian Journal of Education, 6(3), 1-24.

Kim, S.Y., Park, J.Y., Kim, J.C., \& Kang, H.S. (2001). Daehank haksawajung kangyi peong gaje siltaebunsokul tonghan kyoyukupjok peongga mohyung yeongu. Seoul: Ministry of Education \& Human Resources Develpoment. 